\title{
MUCOCELES OF THE APPENDIX- A RETROSPECTIVE STUDY FROM A TERTIARY HEALTH CARE CENTRE.
}

Kamal Malukani, Shirish Nandedkar, Gaurav Malpani, Aparna Khandelwal, Pramod Sakhi

Prasann Kumar Bandi
1. Assistant Professor, Department of Pathology, Sri Aurobindo Institute of Medical Sciences, Indore (M. P.)
2. Professor and Head, Department of Pathology, Sri Aurobindo Institute of Medical Sciences, Indore (M. P.)
3. P. G. Resident, Department of Pathology, Sri Aurobindo Institute of Medical Sciences, Indore (M. P.)
4. P.G. Resident, Department of Pathology, Sri Aurobindo Institute of Medical Sciences, Indore (M. P.)
5. Associate Professor, Department of Radiology, Sri Aurobindo Institute of Medical Sciences, Indore (M. P.)
6. Professor, Department of Pathology, Sri Aurobindo Institute of Medical Sciences, Indore (M. P.)

\section{CORRESPONDING AUTHOR}

Dr. Kamal Malukani,

Assistant professor, Department of Pathology,

Sri Aurobindo Institute of Medical Sciences,

Bhanwarasala, Indore (M. P.)

E-mail: kamal.malukani@yahoo.com,

Ph: $09009427327,07312729046$.

\begin{abstract}
The mucocele of the Appendix is an uncommon cystic lesion characterised by distension of the appendiceal lumen with mucus. The incidence ranges from $0.3 \%$ to $0.7 \%$ of all appendectomied specimens. OBJECTIVE: We report here the demographic, clinical, radiologic, histopathologic, preoperative and postoperative findings and outcome of patients of mucocele of the appendix diagnosed on histopathologic examination. METHODS: We retrospectively analysed the hospital records of all the patients whose appendectomy was done and had a histopathological diagnosis of mucocele of the appendix. RESULTS: A total of 885 patients with the preoperative diagnosis of appendicitis were admitted and surgically treated in our centre from April'2004 to September'2012 and 13 (1.47\%) patients were diagnosed to have mucocele of the appendix on histopathologic examination. Average age of presentation was 53yrs (range 17yrs - 85yrs). 6(46.15\%) patients were male and 7 (53.85\%) were female with M: F ratio 0.86:1. The most common presentation was right lower quadrant pain in 7 (53.85\%) patients. $2(15.38)$ patients presented with generalised abdominal pain, distension of abdomen and loss of appetite while $4(30.77 \%)$ patients were asymptomatic. In preoperative ultrasonographic examination, appendiceal cystic masses were diagnosed in 7 (53.85\%) patients. Histopathologic examination revealed simple mucocele in $7(53.85 \%)$ patients, mucinous cystadenoma in 5 $(38.46 \%)$ and mucinous cystadenocarcinoma in $1(7.69 \%)$ patient. Two patients of mucinous cystadenoma were diagnosed with complication of pseudomyxoma peritonei. No mortality was noted in any of the patient postoperatively and on follow up. CONCLUSION: Mucocele of the appendix is a rare disease and is usually diagnosed on histopathologic examination of Appendectomied specimens. As there is potential for malignant transformation and pseudomyxoma peritonei due to rupture of the mucocele, extensive preoperative evaluation and thorough intraoperative gastrointestinal, ovarian \& peritoneal examination is required.
\end{abstract}

KEY WORDS: Appendix, mucocele, mucinous cystadenoma, mucinous cystadenocarcinoma, pseudomyxoma peritonei

MESH : Appendix, cystadenoma, mucocele, male, female, pseudomyxoma peritonei. 
INTRODUCTION: Appendiceal mucocele, first described by Rokitansky (1); refers to localised or diffuse dilatation of appendiceal lumen by an abnormal accumulation of mucus. It represent $0.3 \%-0.7 \%$ of appendiceal pathology and $8 \%$ of appendiceal tumors (2). Its frequency is higher in females (M: $\mathrm{F}=1: 4$ ) and in people older than 50 yrs of age (3). Mucocele of appendix are difficult to diagnose despite extensive preoperative evaluation. Patients are often asymptomatic and lesions are usually discovered incidentally intraoperatively or postoperatively during histopathological examination (3). Others may present as acute appendicitis or as acute or chronic non specific abdominal pain with or without vomiting.

Mucoceles of appendix can be divided into three categories (4):

1. Simple or Retention mucocele resulting from obstruction of appendiceal outflow and characterised by normal or hyperplastic epithelium with mild luminal dilatation upto $1.0 \mathrm{~cm}$. These constitute $5-25 \%$ of mucoceles.

2. Mucinous cystadenoma - Most common form accounting for 63- 84\% cases of mucoceles. These exhibit epithelial villous adenomatous changes with some degree of epithelial atypia and characterized by marked distension of lumen (upto $6.0 \mathrm{~cm}$.). The neoplastic epithelium is similar to that seen in villous adenomas and adenomatous polyps.

3. Mucinous cystadenocarcinoma - representing $11-20 \%$ of cases. These show glandular stromal invasion and / or presence of epithelial cells in peritoneal implants. The neoplastic epithelium is similar to that seen in adenocarcinoma of colon.

Mucinous cystadenoma and mucinous cystadenocarcinoma may rupture producing fatal entity pseudomyxoma peritonei.

METHOD: This was a unicentric retrospective study, patients with the diagnosis of Appendiceal mucocele were searched from the hospital records. These patients were evaluated for age, sex, symptom duration, major complaint, ultrasonographic and computed tomographic findings, surgical procedures performed, histopathologic diagnosis, complications if any and their follow up outcomes.

INCLUSION CRITERIA: All cases diagnosed as Appendiceal mucocele on Histological examination were included.

EXCLUSION CRITERA: Cases suspected as Appendiceal mucocele on ultrasonography or/ and computed tomography but not proved on histological examination were excluded.

RESULTS: A total of 885 patients with the preoperative diagnosis of appendicitis were admitted and surgically treated in our centre from April' 2004 to September'2012. Out of them 13 ( $1.47 \%$ ) patients were diagnosed to have mucocele of the appendix on histopathologic examination. Average age of the patients in years was 53 yrs (range 17yrs - 85yrs). 6 (46.15\%) patients were male and 7 (53.85\%) were female with $\mathrm{M}: \mathrm{F}$ ratio 0.86: 1 . The duration of the symptoms was between 1 day to 2 months, with a median of 6 days. Right lower quadrant abdominal pain was the most common complaint in $7(53.85 \%)$ patients while $2(15.38 \%)$ patients were admitted with complaints of generalised abdominal pain and loss of appetite. These 2 patients were females and had distension of abdomen with mild to moderate ascitis. Appendiceal mucocele was detected incidentally in $4(30.77 \%)$ patients. Laboratory 
investigations showed leucocytosis in 8 (61.54\%) patients with increased absolute neutrophil count. All other routine biochemical and haematological parameters were within normal limits. Preoperative ultrasonographic examination of the patients revealed appendiceal cystic mass (mucocele) with variable internal echogenicity in 7 (53.85\%) patients, while free fluid was found in abdomen in 2 of these 7 patients. Abdominal CT examination was done in $4(30.77 \%)$ patients ; 2 patients were reported as having appendiceal cystic tumoral mass containing mural calcification. Peritoneal fluid was tapped from 2 patients who had ascitis, and was reported as pseudomyxoma peritonei / mucinous tumor in view of presence of clusters of epithelial cells and stromal fragments in mucinous background.

Appendiceal cystic mass was appreciated by the surgeons in 10 (76.92\%) patients during intraoperative exploration, out of which 2 patients who had ascitis were found to have dense mucinous deposits on appendix, omentum, uterine, ovarian and peritoneal surfaces. Appendix was ruptured in both of these cases. 11 (84.62\%) patients were treated by appendectomy. In 2 patients who had ruptured mucocele with diffuse pseudomyxoma peritonei, caecectomy with omenectomy was performed. As both these patients were postmenopausal females and dense mucinous deposits were present on uterine and ovarian surface, total abdominal hysterectomy with bilateral salpingo-oophorectomy was also done. Both these patients were given early postoperative chemotherapy.

Histopathologic examination revealed simple mucocele in 7 (53.85\%) patients, mucinous cystadenoma in $5(38.46 \%)$ patients, mucinous cystadenocarcinoma in $1(7.69 \%)$ patient. In the 7 patients with preoperative ultrasonographic diagnosis of appendiceal cystic masses (mucocele), histopathologic examination showed simple mucocele in 3 patients and mucinous cystadenoma in 4 patients. In 1 patient diagnosed as mucinous cystadenocarcinoma, right colectomy was done subsequently. No peritoneal or adjacent organ involvement was seen in this patient and no lymph node metastasis was seen.

In both the patients with pseudomyxoma peritonei, ruptured primary mucinous cystadenoma of appendix was diagnosed on histopathology examination. Pools of mucin were also seen on the surface of both ovaries, uterus, peritoneum and omentum with very few benign looking epithelial cells. Ovarian tumor was ruled out in both these patients.

No postoperative morbidity or mortality occurred. Average postoperative length of hospital stay was 4.6 ( 2 - 10) days. At postoperative follow up of two patients of pseudomyxoma peritonei and one patient of mucinous cystadenocarcinoma ; physical examination, abdominal sonography, carcino-embryonic antigen (CEA) and other laboratory investigations were done twice a year. No tumor was detected in any of the cases on follow up for two years. Long term follow up is required to access the final outcome.

DISCUSSION: Present study showed much higher incidence of appendiceal mucocele $(1.47 \%)$ in appendectomied specimens as compared to $0.3-0.6 \%$ reported in previous studies $(2,5)$. However increased incidence $(2.01 \%)$ of appendiceal mucocele from some tertiary health care centres have also been reported ( 6 ). Female preponderance was seen in our study with $\mathrm{M}: \mathrm{F}$ ratio $0.86: 1$, which is much lower as compared to reported in some previous studies $(3,5)$. However some recent reports show a male predominance (2:1), suggesting that the gender distribution of appendiceal mucoceles is changing (7). Average age of presentation was 53 years which was in concordance to that reported previously $(3,5)$ that is more than 50yrs of age.

In our study 7 (53.85\%) patients presented with right lower quadrant abdominal pain while $2(15.38 \%)$ with generalised abdominal pain, abdominal distension \& loss of appetite. In 
remaining 4 (30.77\%) patients, Appendiceal mucocele was an incidental finding. In a study done by Muthukumaran Rangarajan et al (7), out of 9 patients, 6 ( $66.67 \%$ ) presented with pain localised to right lower quadrant of abdomen while $2(22.22 \%)$ with palpable mass. S. Yakan et al (5) reported right lower quadrant abdominal pain in 8 (89\%) and generalised abdominal pain in $1(11 \%)$ out of 9 patients.

Our study revealed leucocytosis in $8(61.54 \%)$ out of 13 patients. In a similar study by S. Yakan et al (5), leucocytosis was seen in $5(55.56 \%)$ out of 9 patients. In our study preoperative ultrasonographic examination showed appendiceal cystic mass (mucocele) in 7 (53.85\%) patients, while free fluid was found in abdomen in $2(15.38 \%)$ patients. Abdominal CT examination was done in $4(30.77 \%)$ patients; 2 (15.38\%) patients were reported as having appendiceal cystic tumoral mass containing mural calcification. Kemal Karakaya et al (8) reported in their study that USG and CT examination showed appendiceal cystic mass with peripheral enhancement in $3(60 \%)$ out of 5 patients, free fluid in $1(20 \%)$ patient and in one patient CT / USG was not done. In the study of Muthukumaran Rangarajan et al (7), USG was able to diagnose cystic appendiceal mass in $6(66.67 \%)$ patients and CT revealed mucocele in $2(22.22 \%)$ out of 9 cases. While in the study of S. Yakan et al (5), appendiceal cystic mass was diagnosed in $4(44.5 \%)$ patients on USG and in $1(11 \%)$ patient on CT examination.

Simple appendectomy was done in $10(76.92 \%)$ patients in present study, in 2(15.38\%) patients caecectomy and in $1(7.69 \%)$ patient hemicolectomy was done. In the study of S.Yakan et al (5), appendectomy was done in $6(67 \%)$ and right hemicolectomy in 2(22\%) patients. Kemal Karakaya et al (8) have reported simple appendectomy in 3( $60 \%$ ) and caecectomy in 2 $(40 \%)$ patients. Present study revealed higher incidence of simple retention mucocele as compared with previous studies. Histopathologic examination showed simple mucocele in $7(53.85 \%)$, mucinous cystadenoma in $5(38.46 \%)$ patients and mucinous cystadenocarcinoma in $1(7.69 \%)$ patient. S Yakan et al(5) have reported simple mucocele in 2(22\%), mucinous cystadenoma in $4(45 \%)$ and mucinous cystadenocarcinoma in 3(33\%) cases. Kemal karakaya et al(8) have reported four cases of mucinous cystadenoma and one case of simple mucocele.

Different theories had been postulated in literature about mucocele origin. The first, postulated by Neeslund (9), is mechanical and starts from an obstacle (diverticulitis, inflammation, polyps) at the base of appendix, which gives an accumulation of mucus inside the appendix, increasing volume and consequently causing rupture. Another theory is nervous; hyperincretion of mucus and muscular paralysis could cause the changement of appendix in a cyst (9). According to Higa classification we know retention cysts, caused by mucin accumulation and dilations due to hyperproduction of mucus by an appendicular benign or malignant neoplasia (10). In this last condition it is important to specify the nature of the malignancy because gelatinous mass cells have an autonomic growth maintaining function of mucus secretion (11).

USG, CT and colonoscopic examinations can facilitate preoperative diagnosis of appendiceal mucocele (12-15). Ultrasound is the first line diagnostic modality for patients with acute abdominal pain or mass. Different sonographic findings of appendiceal mucocele and acute appendicitis have been described (16-18). Outer diameter of appendix $15 \mathrm{~mm}$ or more in USG examination has been described as the threshold for the diagnosis of appendiceal mucocele with a sensitivity of $83 \%$ and a specificity of $92 \%$ (16), while for acute appendicitis it has been established as 6mm (19). USG examination revealed appendiceal cystic mass in 7 of our patients. CT is the modality of the choice in cases of appendiceal mucocele because of its ability to detect the anatomic location of mass and tissue characteristics. Mural curvilinear calcification 
aids considerably in the diagnosis but occurs in less than $50 \%$ cases (20). USG and CT findings are not specific and the differential diagnosis should be established with other pathologies such as carcinoid, lymphoma, mesenteric cysts and ovarian masses $(2,21)$. Fine needle aspiration of the appendiceal mucocele is generally avoided because of fear that puncture of a distended viscus will lead to localized or diffuse pseudomyxoma peritonei (17). Colonoscopy in patients with abdominal pain is a useful tool for determination of mucocele $(22,23)$. Generally an elevation of the orifice of the appendix is seen. A yellowish mucous discharge would be visible from appendiceal orifice during colonoscopy. 'Volcano sign' is appendiceal orifice in the centre of a firm mound covered by normal mucosa or lipoma like submucosal mass. Colonoscopy is also important for the diagnosis of synchronous and metachronous colon tumor. Elevated CEA levels have been described in neoplastic mucoceles ( 24)

The spontaneous and surgery induced complications of appendiceal mucocele include intestinal obstruction, intussusceptions $(21)$, intestinal bleeding $(13,25)$, fistula formation $(15)$ and volvulus ( 26 ). The worst complication is pseudomyxoma peritonei. The preoperative diagnosis that distinguishes appendiceal mucocele from acute appendicitis is essential for the best choice of surgical approach (open Vs laproscopic) to prevent peritoneal dissemination of mucin producing epithelial cells and perform the appropriate surgery $(16,27)$. It was thought that only mucinous cystadenocarcinomas lead to pseudomyxoma peritonei (3). However other authors believe pseudomyxoma peritonei can complicate either benign or malignant mucoceles, although pseudomyxoma peritonei from the former would carry a better prognosis $(10,28,29)$. No reports of lymphatic or hematogenous spread of mucinous cystadenocarcinoma are found in the literature.

Most acknowledge that pseudomyxoma peritonei predominantly originates in the appendix in men and increasingly evidence suggests a similar site of origin in females $(30,31)$. In women synchronous ovarian and appendiceal disease is common, and Pseudomyxoma peritonei appears more prevalent. However immunohistochemistry and molecular genetic techniques support the hypothesis that in the majority of women, the ovarian tumor is metastatic from a perforated appendiceal mucinous tumor (32 - 35).

Concomitant cases of appendiceal mucocele and colon adenocarcinoma have been described with an incidence of $19.5 \%-21.4 \%(10,28,36)$. In our study no such concomitance was noted. The progression of mucinous cystadenoma to mucinous cystadenocarcinoma has not been proved yet, but it is suggested.

Dhage- Ivatury and Sugarbaker (37) have suggested simple appendectomy as the choice of treatment for patients with benign mucocele that has negative margins of resection and without perforation. No long term follow up is needed for these patients. In patients having perforated mucocele with positive margins of resection and negative appendiceal lymph nodes, caecectomy / cytoreductive surgery (CRS) / Heated intraperitoneal chemotherapy ( HIIC ) and early postoperative intraperitoneal chemotherapy (EPIC ) should be performed. Long term follow - up is also obligatory. In patients having perforated mucocele with positive margins of resection and positive appendiceal lymph nodes, right hemicolectomy / CRS / HIIC and EPIC should be performed. Long term follow-up is also obligatory in these patients.

The 5- year survival rate for simple or benign neoplastic mucocele after appendectomy ranges from $91 \%$ to $100 \%$, but recurrences as pseudomyxoma peritonei and metachronic colonic neoplasms causing mortality can be seen $(3,38,39)$. Cystadenocarcinoma without peritoneal or adjacent organ involvement also show good outcome after surgical resection, but 
if it progresses to pseudomyxoma peritonei, 5 yr survival is $25 \%$, with most deaths attributed to intestinal obstruction and renal failure (40).

CONCLUSION: Appendiceal mucocele is a relatively uncommon pathology seen in people older than 50 yrs of age with female preponderance. Surgical treatment of appendiceal mucocele is mandatory because of the potential for malignant transformation and pseudomyxoma peritonei due to rupture of the mucocele itself. Preoperative suspicion and diagnosis of appendiceal mucocele are important. Appendiceal mucocele should be considered in patients presenting as right lower quadrant abdominal pain or diffuse abdominal pain. Ultrasonography and computed tomography are useful tools for the diagnosis of appendiceal mucocele. Accurate preoperative diagnosis is a major component for optimal management, to minimize intraoperative and post operative complications. Extreme care must be taken while handling the tissue during operation. Intraoperative exploration of the entire gastrointestinal tract and ovaries in females should be done. All gross peritoneal implants should be removed and examined for presence of epithelial atypia for diagnostic and prognostic purposes.

\section{REFERENCES:}

1. Rokitansky CF. A manual of pathological anatomy. Philadelphia (PA) : Blanchard \& Lea; 1855. P 89.

2. Ruiz-Tovar J, Teruel DG, Castiñeiras VM, Dehesa AS, Quindós PL, Molina EM. Mucocele of the appendix. World J Surg. 2007; 31: 542-548.

3. Aho AJ, Heinonen R, Lauren P. Benign and malignant mucocele of the appendix. Acta Chir Scand. 1973; 139 (4) : 392-400.

4. Qizilbash AH. Mucoceles of the appendix. Their relationship to hyperplastic polyps, mucinous cystadenomas and cystadenocarcinomas. Arch Pathol Lab Med. 1975; 99: 548 $-555$.

5. Yakan S, Caliskan C, Uguz A, Korkut MA, Coker A. A retrospective study on mucocele of the appendix presented with acute abdomen and acute appendicitis. Hong Kong J Emerg Med 2011; 18 : 144 - 149.

6. Karakaya K, Barut F, Emre AU, Ulcan HB, Cakmak GK, Irkorucu O, Tascilar O, Ustundag Y, Comert M. Appendiceal Mucocele : Case reports and review of current literature. World J Gastroenterol 2008; 14 (14) : 2280 - 2283.

7. Muthukumaran Rangarajan, Chinnusamy Palanivelu, Alfie Jose Kavalakat, Ramakrishnan Parthasarathi. Laparoscopic appendectomy for mucocele of the appendix : report of 8 cases. Indian J Gastenterol. 2006; 25 : 256 - 57.

8. Kemal Karakaya, Figen Barut, Ali Ugur Emre, Hamdi Bulent Ucan, Guldeniz Karadeniz Cakmak, Oktay Irkorucu, Oge Tascilar, Yucel Ustundag, Mustufa comert. Appendiceal Mucocele : Case reports and review of current literature. World J Gastroenterol. 2008 April 14; 14 (14) : 2280 - 83.

9. Alberti P, Bonera A, Antoci G e Bianchi P. II mucocele appendicolare. Min Chir. 1993; 48: 865-870.

10. Higa E, Rosai J, Pizzim Bono CA, Wise L. Mucosal hyperplasia, mucinous cystadenoma and mucinous cystadenocarcinoma of appendix. A re-evaluation of appendiceal mucocele. Cancer. 1973; 32:1525-1541. 
11. Woodruff R, Mac Donald JR. Benign and malignant cystic tumors of the appendix. Surg Gynecol Obstet.1950; 71: 750-753.

12. Pitiakoudis M, Tsaroucha AK, Mimidis K, Polychronidis A, Minipoulos G, Simipolous C. Mucocele of the appendix : a report of five cases. Tech Coloproctol. $2004 ; 8: 109-112$.

13. Qualia CM, Drugas GT, Jones LT, Rossi TM. Colonoscopic diagnosis of an appendiceal mucocele. J Pediatr Gastroenterol Nutr. 2007; 45 : 145 - 146.

14. Minagawa M, Ishikawa H, Date K, Kosugi S, Hatakeyama K, Endo K, Kimura K, Fukuda F. Mucus outflow from the appendiceal orifice due to an appendiceal mucocele. Gastrointest Endosc. 2001; 53: 493.

15. Nakao A, Sato S, Nakashima A, Nabeyama A, Tanaka N. Appendiceal mucocele of mucinous cystadenocarcinoma with a cutaneous fistula. J Int Med Res. 2002; 30 : 452 456.

16. Lein WC, Huang SP, Chi CL, Liu KL, Lin MT, Lai TI, Liu YP, Wang HP. Appendiceal outer diameter as an indicator for differentiating appendiceal mucocele from appendicitis. Am J Emerg Med. 2006; 24: 801 - 805.

17. Pickhardt PJ, Levy AD, Rohrmann CA Jr, Kende AI. Primary neoplasms of the appendix : radiologic spectrum of disease with pathologic correlation. Radiographics. 2003; 23 : $645-662$.

18. Sasaki K, Ishida H, Komatsuda T, Suzuki T, Konno K, Ohtaka M, Sato M, Ishida J, Sakai T, Watanabe S. Appendiceal mucocele : sonographic findings. Abdom Imaging. 2003; 28 : $15-18$.

19. Birnbaum BA, Wilson SR. Appendicitis at the millennium. Radiology. 2000; $215: 337$ 348.

20. Madved D, Mindelzum R, Jeffrey RB Jr. Mucocele of the appendix : Imaging finding. AJR Am J Roentgenol. 1992; 159:69 -72.

21. Cois A, Pisanu A, Pilloni L, Uccheddu A. Intussusception of the appendix by mucinous cystadenoma. Report of a case with an unusual clinical presentation. Chir Ital. 2006; 58 : $909-910$.

22. Zanati SA, Martin JA, Baker JP, Streutker CJ, Marcon NE. Colonoscopic diagnosis of mucocele of the appendix. Gastrointest Endosc. 2005; 62: $452-456$.

23. Watanabe T, Yoshikawa I, Kihara Y, Kume K, Otsuki M. Appendiceal mucocele. Gastrointest Endosc. 2003; 58 : 909 - 910.

24. Soweid AM, Clarkston WK, Andrus CH, Janney CG. Diagnosis and management of appendiceal mucoceles. Dig Dis. 1998; 16(3) : 183 - 186.

25. Lakatos PL, Gyori G, Halasz J, Fuszek P, Papp J, Jaray B, Lukovich P, Lakatos L. Mucocele of the appendix : an unusual cause of lower abdominal pain in a patient with ulcerative colitis. A case report and review of literature. World J Gastroenterol. $2005 ; 11: 457$ 459.

26. Rudloff U, Malhotra S. Volvulus of an appendiceal mucocele : report of a case. Surg Today. 2007; $37: 514-517$.

27. Gonzalez Moreno S, Shmookler BM, Sugarbaker PH. Appendiceal mucocele. Contraindication to laproscopic appendectomy. Surg Endosc. 1998; 12: 1177 - 1179.

28. Kim SH, Lim HK, Lee WJ, Lim JH, Byun JY. Mucocele of the appendix: ultrasonographic and CT findings. Abdominal imaging. 1998; 23: 292 - 296.

29. Balthazar EJ, Megibow AJ, Gordon RB, Whelan CA, Hulnick D. Computed Tomography of the abnormal appendix. J Comput Assist Tomogr. 1988; 12: 595 - 601. 
30. Mukherjee A, Parvaiz A, Cecil TD, Moran BJ. Pseudomyxoma Peritonei usually originates from the appendix: a review of the evidence. Eur J Gynaecol Oncol. 2004; 25: 411-414.

31. Sherer DM, Abulafia 0, Eliakim R. Pseudomyxoma Peritonei: a review of current literature. Gynaecol Obstet Invest. 2001; 51: 73-80.

32. Ronnett BM, Shmookler BM, Diener - West M, Sugarbaker PH, Kurman RJ. Immunohistochemical evidence supporting the appendiceal origin of pseudomyxoma peritonei in women. Int J Gynecol Pathol. 1997; 16: 1-9.

33. Szych C, Staebier A, Connolly DC, Wu R, Cho KR, Ronnett BM. Molecular genetic evidence supporting the clonality and appendiceal origin of pseudomyxoma peritonei in women. Am J Pathol. 1999; 154: 1849-1855.

34. Chuaqui RF, Zhuang Z, Emmert - Buck MR, Bryant BR, Nogales F, Tavassoli FA, Merino MJ. Genetic analysis of synchronous mucinous tumors of the ovary and appendix. Hum Pathol. 1996; 27: 165 -171.

35. Guerrieri C, Franlund B, Fristedt S, Gillooley JF, Boeryd B. Mucinous tumors of the vermiform appendix and ovary, and pseudomyxoma peritonei; histogenetic implications of cytokeratin 7 expression. Hum Pathol. 1997; 28: 1039 - 1045.

36. Fujiwara T, Hizuta A, Iwagaki H, et al. Appendiceal mucocele with concomitant colonic cancer. Report of two cases. Dis Colon Rectum. 1996; 39: 232 - 236.

37. Dhage - Ivatury S, Sugarbaker PH. Update on the surgical approach to mucocele of the appendix. J Am Coll Surg. 2006; $202: 680$-684.

38. Carr NJ, McCarthy WF, Sobin LH. Epithelial non- carcinoid tumors and tumor like lesions of the appendix. A clinicopathological study of 184 patients with a multivariate analysis of prognostic factors. Cancer. 1995; 75: 757 - 768.

39. Smith JW, Kemeny N, Caldwell C, Banner P, Sigurdson E, Huvos A. Pseudomyxoma peritonei of appendiceal origin. The Memorial - Sloan Kettering Cancer Center experience. Cancer. 1992; 70 (2) : 396 - 401.

40. Isaac KL, Warshauer DM. Mucocele of the appendix : computed tomographic, endoscopic and pathologic correlation. Am J Gastroenterol. 1992; 87 (6) : 787 - 789.

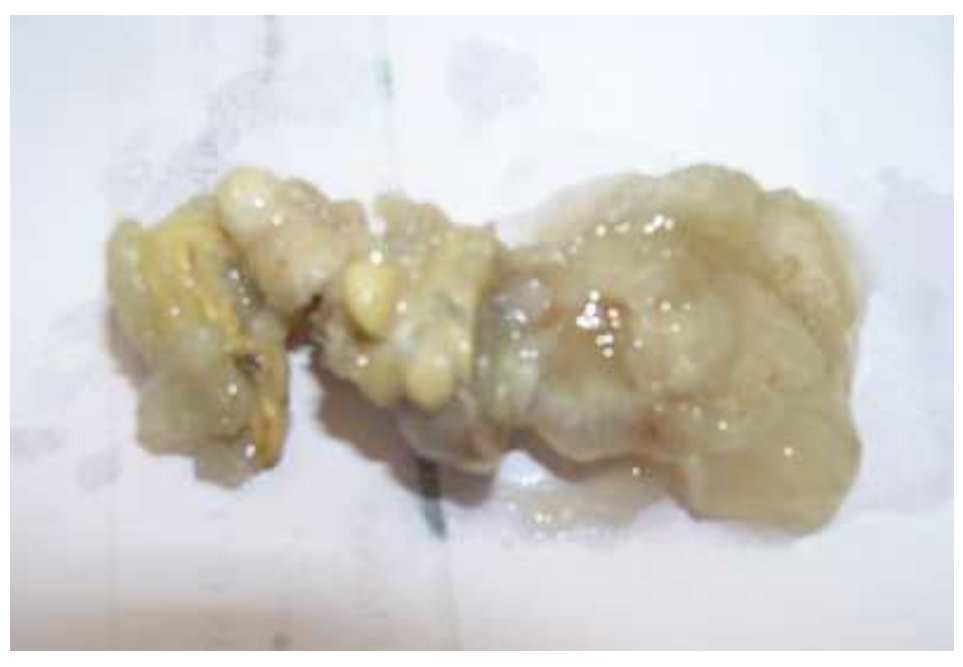

Fig 1 - Gross appearance of Mucocele of the Appendix, cystically dilated with abundant mucin and ruptured. 


\section{ORIGINAL ARTICLE}

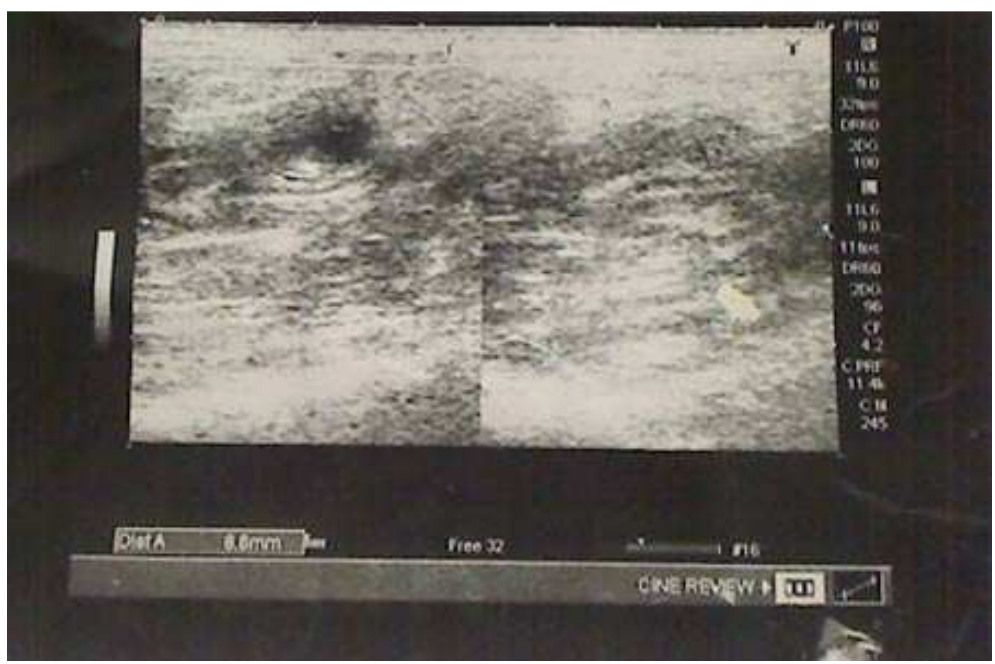

Fig 2 - High resolution Ultrasonography of right iliac fossa showing lobulated thick walled hypoechoic blind loop.

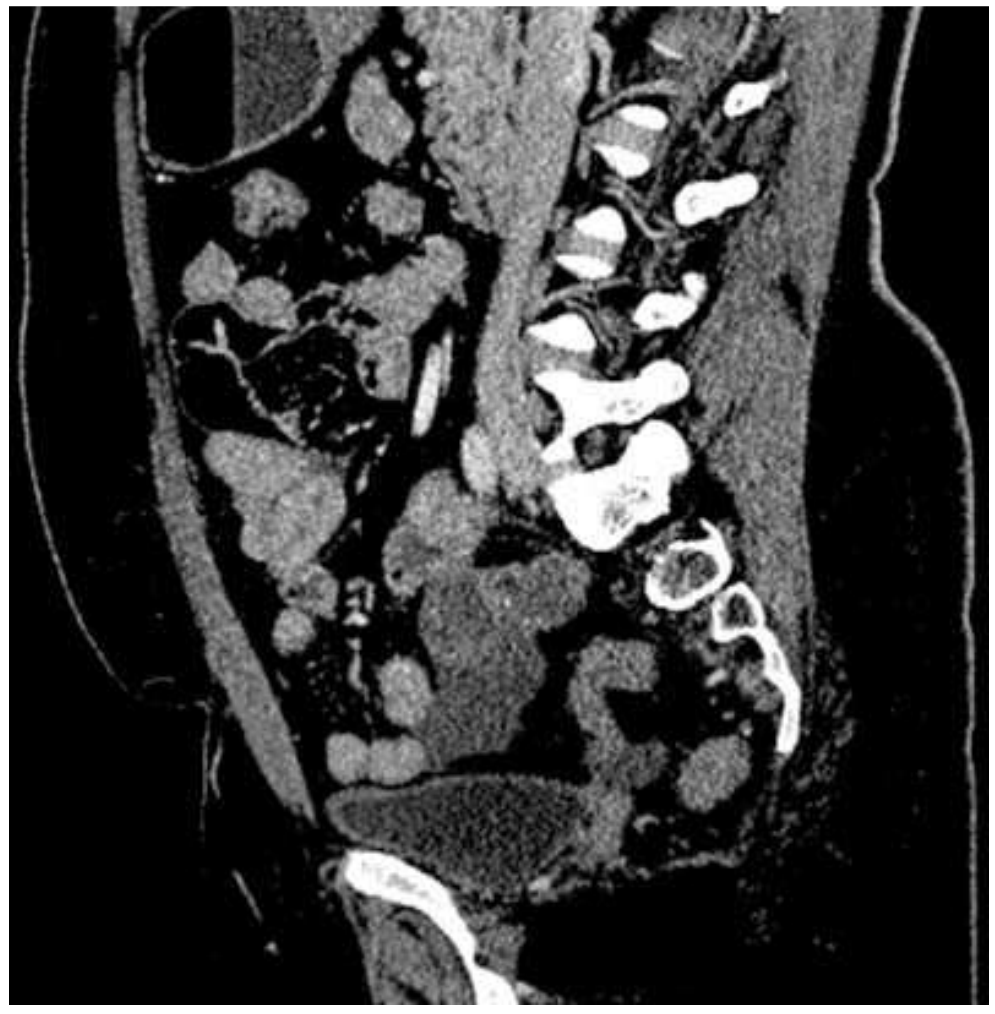

Fig 3 - Abdominal computed tomography, sagittal section - lobulated blind loop hypodense lesion showing wall calcification with localized fluid just below blind loop suggesting ruptured Mucocele of Appendix. 


\section{ORIGINAL ARTICLE}

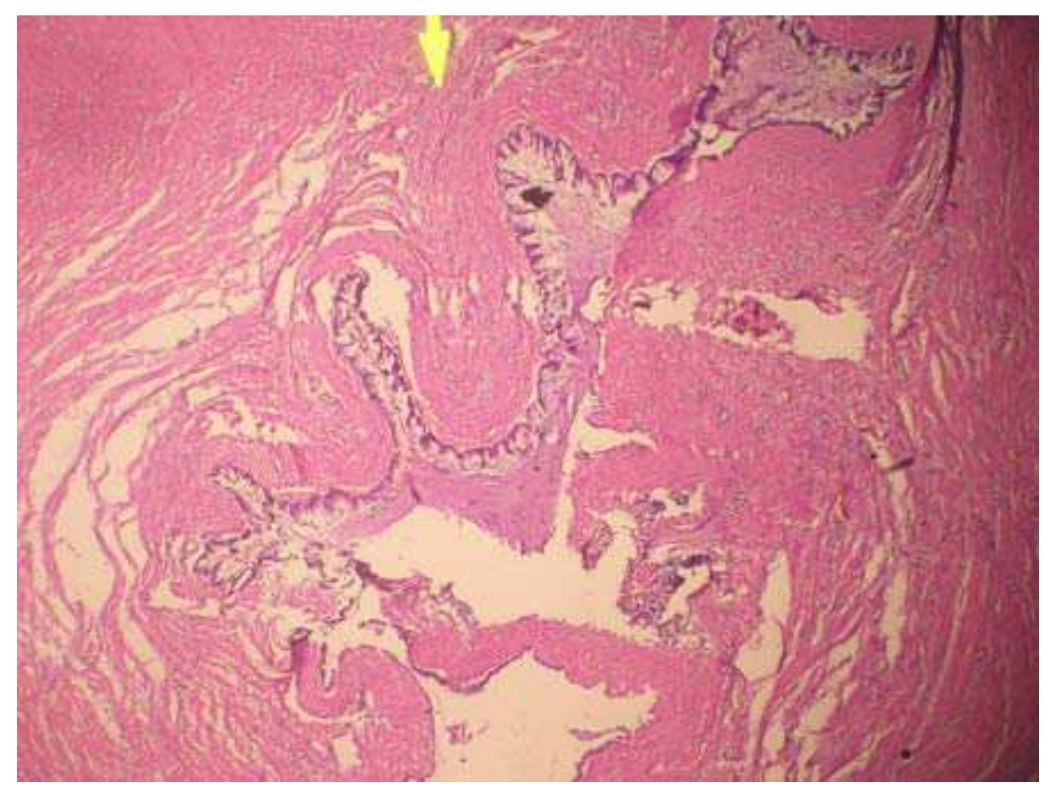

Fig 4 - Microphotograph showing Mucinous cystadenoma of the Appendix; wall is lined by mucin secreting epithelium with mucin in the lumen (H \& E).

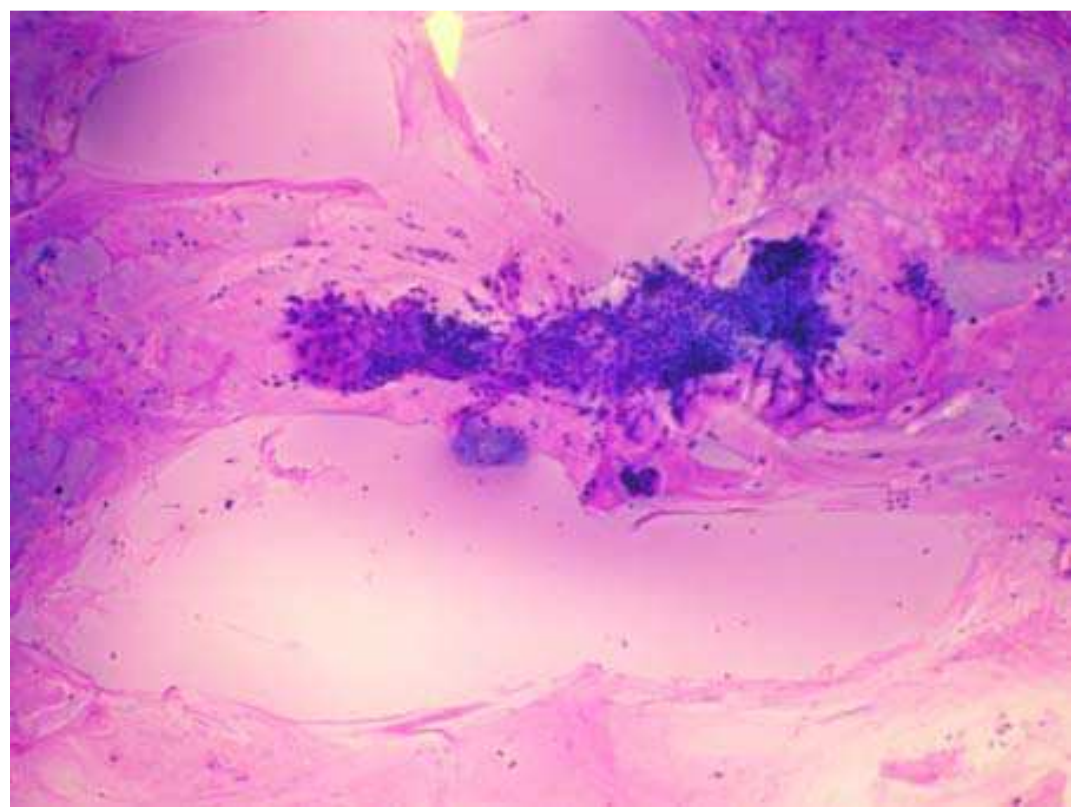

Fig 5 - Pseudomyxoma Peritonei - Ascitic fluid cytology showing mucinous material with groups of epithelial cells (Geimsa). 